\title{
Aplicación del modelo Aquacrop para un cultivo de maíz (Zea mays $L$ )
}

\author{
Application of the Aquacrop model for a corn crop (Zea mays L.)
}

\section{Aplicação do modelo Aquacrop para cultivo do milho (Zea mays L.)}

\author{
Ramos Cantillo José Alexander ${ }^{1}$, Becerra Vélez Eduardo ${ }^{1}$, \\ Cárdenas Hernández Julián Fernando ${ }^{2}$ y Jiménez Pizarro Rodrigo ${ }^{3}$ \\ ${ }^{1}$ Ingenieros Agrónomos, Universidad de los Llanos. \\ ${ }^{2}$ Ingeniero Agrónomo, MSc. Docente Universidad de los Llanos \\ ${ }^{3}$ Ingeniero Químico, PhD, Universidad Nacional de Colombia \\ jfcardenas@unillanos.edu.co
}

Recibido 19 de febrero 2019, Aceptado 2 de octubre 2019

\section{RESUMEN}

En la producción agrícola se usan una amplia cantidad de modelos descriptivos que buscan predecir la productividad, desarrollo y comportamiento de los cultivos bajo diferentes escenarios de manejo agronómico, factores edáficos, condiciones ambientales y características genotípicas. Entre dichos modelos uno de los más utilizados actualmente y ampliamente distribuidos se encuentra AquaCrop que es descriptivo y que simula la biomasa y el rendimiento potencial cosechable de un cultivo en respuesta a la disponibilidad de agua. Este trabajo se realizó con el propósito de medir el crecimiento y comprender el comportamiento de los diferentes órganos del maíz comercial hibrido BM709, en lo referente a su acumulación de materia seca bajo las condiciones de Altillanura. Dicha evaluación consto de 10 muestreos de medidas biométricas en plantas fijas, y que fueron monitoreadas por la torre de Eddy covariance, para recibir la información de las condiciones climáticas de la zona. Para las simulaciones del comportamiento maíz, se utilizó la versión 5.0 del AquaCrop, la cual fue adaptada a las condiciones climatológicas y suelos de la zona para mayor confiabilidad en la estimación de las variables de estudio: materia seca total de la planta, área foliar e índices de crecimiento. El análisis de desarrollo del cultivo en el software AquaCrop presento una representatividad entre lo simulado y lo real superior al $90 \%$ generando de esta manera un patrón de 
confiabilidad en la toma de decisiones referidas a maximizar los rendimientos del cultivo de maíz. Además logró mostrar que la producción total de materia seca es el resultado de la eficiencia cuando el cultivo intercepta y utiliza la radiación solar disponible durante la fase de crecimiento, la cual en condiciones de Altillanura es buena por los factores ambientales y climáticos, entre ellos el $\mathrm{CO}_{2}$, radiación, temperatura y precipitación, es de anotar que a los $40^{\circ} \mathrm{C}$, cuando las condiciones hídricas, radiación, manejo agronómico sean adecuadas el maíz podría mantener o aumentar su productividad por la alta concentración de $\mathrm{CO}_{2}$ en el ambiente y su capacidad de alcanzar una tasa de crecimiento del cultivo eficiente, con un índice de área foliar crítico.

Palabras clave: Fisiología, cereales, modelos de simulación.

\begin{abstract}
In agricultural production, a large number of descriptive models are used that seek to predict the productivity, development and behavior of crops under different agronomic management scenarios, edaphic factors, environmental conditions and genotypic characteristics. Among these models, one of the most widely used and widely distributed is AquaCrop, which is descriptive and simulates the biomass and potential crop yield of a crop in response to water availability. This work was carried out with the purpose of measuring growth and understanding the behavior of the different organs of BM709 hybrid commercial corn, in relation to its accumulation of dry matter under the conditions of the plain. This evaluation consisted of 10 samples of biometric measurements in fixed plants, and which were monitored by the Eddy covariance tower, to receive information on the climatic conditions of the area. For the simulations of the corn behavior, version 5.0 of the AquaCrop was used, which was adapted to the climatic conditions and soils of the area for greater reliability in the estimation of the study variables: total dry matter of the plant, foliar area and growth rates The analysis of crop development in the AquaCrop software presented a representativeness between the simulated and the real over $90 \%$, generating in this way a pattern of reliability in the decision-making process related to maximizing the yields of the corn crop. Total dry matter production is the result of efficiency when
\end{abstract}


the crop intercepts and uses the available solar radiation during the growth phase, which under of the plain conditions is good due to environmental and climatic factors, including $\mathrm{CO}_{2}$, radiation, temperature and precipitation, it should be noted that at $40^{\circ} \mathrm{C}$, when water conditions, radiation, agronomic management are adequate, corn could maintain or increase its productivity due to the high concentration of $\mathrm{CO}_{2}$ in the environment and its ability to reach a crop growth rate efficient, with an index of critical leaf area.

Keywords: Physiology, cereals, simulation models.

\section{RESUMO}

Na produção agrícola, utiliza-se um grande número de modelos descritivos que buscam prever a produtividade, o desenvolvimento e o comportamento das culturas em diferentes cenários de manejo agronômico, fatores edáficos, condições ambientais e características genotípicas. Entre esses modelos, um dos mais amplamente utilizados e amplamente distribuídos é o AquaCrop, que é descritivo e simula a biomassa e o potencial rendimento de uma cultura em resposta à disponibilidade de água. Este trabalho foi realizado com o objetivo de medir o crescimento e entender o comportamento dos diferentes órgãos do milho comercial híbrido BM709, em relação ao acúmulo de matéria seca nas condições da planicie, que consistiu em 10 amostras de medidas biométricas em plantas fixas e monitoradas pela torre de covariância por redemoinho, para receber informações sobre as condições climáticas da área. Para as simulações do comportamento do milho, foi utilizada a versão 5.0 do AquaCrop, a qual foi adaptada às condições climáticas e solos da área para maior confiabilidade na estimação das variáveis de estudo: matéria seca total da planta, área foliar e taxas de crescimento $A$ análise do desenvolvimento da safra no software AquaCrop apresentou uma representatividade entre o simulado e o real acima de $90 \%$, gerando, dessa forma, um padrão de confiabilidade no processo de tomada de decisão relacionado à maximização do rendimento da safra de milho. A produção total de matéria seca é o resultado da eficiência quando a cultura intercepta e utiliza a radiação solar disponível durante a fase de crescimento, o que nas condições da planicie é bom 
devido a fatores ambientais e climáticos, incluindo $\mathrm{CO}_{2}$, radiação, temperatura e precipitação, deve-se notar que a $40^{\circ} \mathrm{C}$, quando as condições da água, radiação, manejo agronômico são adequadas, o milho pode manter ou aumentar sua produtividade devido à alta concentração de $\mathrm{CO}_{2}$ no ambiente e à sua capacidade de atingir uma taxa de crescimento da cultura eficiente, com um índice de área foliar crítica.

Palavras-chave: Fisiologia, cereais, modelos de simulação.

\section{INTRODUCCIÓN}

El cambio climático es uno de los retos que se deben afrontar para alcanzar un desarrollo sostenible, la alteración de estas condiciones ambientales están íntimamente ligada al modelo de producción y consumo, en Colombia al igual que muchos países se han observado modificaciones en precipitación y temperatura, reflejo de fenómenos globales y de las particularidades de la geografía colombiana, los cuales han tenido repercusiones sobre la producción de algunos cultivos agrícolas; por tal motivo, uno de los objetivos de la agroclimatología ha sido analizar las interacciones del sistema atmosfera-planta-agua-suelo, así como detectar y definir los factores que limitan la producción agropecuaria. También lo es la predicción del rendimiento de los cultivos y los futuros comportamientos de las variables que pueden afectar positiva o negativamente a la planta, generando así, la necesidad de una herramienta útil para agricultores que les permita tomar decisiones acertadas en las diferentes etapas del cultivo y evitar pérdidas en su producción (Fernández, 2013). Actualmente, se están utilizando herramientas con el fin de minimizar el grado de incertidumbre y tener la respuesta sobre el comportamiento de los cultivos en condiciones climáticas de mucha variabilidad (Ojeda et al., 2013). Las discusiones en torno a la productividad del maíz deben siempre tener como trasfondo una serie de problemas como lo son la creciente demanda global por este alimento, y que su expansión se ha limitado de las fronteras agrícolas por las diferentes condiciones climáticas como son: disponibilidad de agua, distribución y duración lluvias en la Altillanura, y en todo el territorio Colombiano, afectando el desarrollo y rendimiento de cultivos como el 
maíz; por tal motivo es importante que se implementen estrategias para que faciliten la adaptación de la agricultura a estos cambios que están experimentando ahora y que seguirán en el futuro (Bello et al., 2013).

De acuerdo a lo anterior, en la producción agrícola se usan una amplia cantidad de modelos descriptivos que buscan predecir la productividad, desarrollo y comportamiento de los cultivos bajo diferentes escenarios de manejo agronómico, factores edáficos, condiciones ambientales y características genotípicas. Entre dichos modelos uno de los más utilizados actualmente y ampliamente distribuidos se encuentra el desarrollado por la $\mathrm{FAO}$, AquaCrop que es un modelo descriptivo que simula la biomasa y el rendimiento potencial cosechable de un cultivo en respuesta a la disponibilidad de agua. AquaCrop es un modelo que estima el rendimiento de cultivos herbáceos donde se incluyen forrajes, vegetales, frutas, aceite, raíces y tubérculos (Fernández, 2013). Considerando que la Altillanura Colombiana es una inmensa sabana con una superficie aproximada de 250.000 $\mathrm{km}^{2}$, cuyas condiciones restrictivas del suelo han limitado el desarrollo agrícola, pero bajo el escenario actual se han superado algunos problemas y se estima que en el corto plazo se incorporen importantes áreas a las a la siembra de cultivos de forma tecnificada como el maíz (Etter et al., 2010.

En la zona de Altillanura, se ha observado un crecimiento en el número de hectáreas del cultivo tecnificado de maíz tecnificado y se espera que esta área aumente para año 2022 a cerca de 60.000. con el cambio de uso de la tierra, como área cultivable en grandes extensiones, se genera un aumento en los cambios de los regímenes climáticos y flujos netos de $\mathrm{CO}_{2}$ en la atmosfera que se enmarcan en una problemática regional y mundial, esto supone en el tiempo un gasto económico. Ante estos problemas es fundamental la incorporación de herramientas tecnológicas y el desarrollo de estrategias de manejo que permitan una intensificación de la producción de maíz, incrementando los rendimientos por unidad de superficie, utilizando eficientemente los recursos que requiere el cultivo (radiación solar, agua y nutrientes, entre otros), pero al mismo tiempo, logrando una reducción de los efectos negativos sobre el ambiente que se derivan del proceso de 
producción (FENALCE, 2011). Se ha establecido que existe un rendimiento del maíz tecnificado económicamente alcanzable que está en el orden del $80 \%$ del rendimiento potencial, que para la zona de Altillanura varia de 7.0-10.5 Ton/ha rendimientos que se encuentran asociados a las condiciones climáticas de la región y los manejos aplicados al cultivo (Bello et al., 2013).

La conversión de ecosistemas naturales a cultivos, no solo modifica los patrones de acumulación de biomasa de las plantas, también cambia el intercambio de agua y energía entre la superficie y la atmósfera. El cambio en el uso y cobertura de la tierra afecta la fenología de la vegetación, modifica propiedades biofísicas de la superficie (rugosidad y albedo), además de alterar el ciclo biogeoquímico del ecosistema (Corbin et al., 2010). El rol de los cultivos en el ciclo de carbono depende en gran medida del tipo de suelo, cultivo, condiciones meteorológicas, prácticas agrícolas y para el caso del cambio de uso del suelo, de la cobertura sustituida (Béziat et al., 2009; Saunders et al., 2012; Corbin et al., 2010).

AquaCrop desarrollado por la $\mathrm{FAO}$, es un modelo enfocado principalmente a la simulación del crecimiento de la planta y producción de biomasa cosechable en respuesta al agua disponible (Raes et al., 2009), tiene la ventaja de ser relativamente sencillo en comparación con otros modelos de simulación de rendimientos, por lo que con sólo calibrar algunos parámetros se obtienen resultados similares a la realidad, por lo que se facilitó adaptarlo a las condiciones colombianas en comparación a otros modelos, además su plataforma del software resulta ser de fácil manejo (Bello, et al, 2013). El motor de este modelo es el agua en donde la transpiración es trasladada dentro de la biomasa por medio de un parámetro denominado la productividad de agua que es la relación entre la biomasa producida en un metro cuadrado por un milímetro transpirado (Fernández, 2013). Puede usarse como herramienta en para analizar escenarios agrícolas en ciclos y localidades diferentes (Hsiao et al., 2009; Steduto et al., 2009). Este modelo determinístico y para su manejo y aplicación no requiere de muchos conocimientos en sistemas, además es simple sin perder exactitud porque usa un número reducido de parámetros en comparación con otros modelos biofísicos comerciales y se ha 
calibrado con buen ajuste y resultados experimentales en varias regiones agrícolas del mundo (Raes et al., 2009).

La estructura de AquaCrop se describe en cuatro aspectos fundamentales (Steduto et al., 2009): 1- Realiza el cómputo separado de la transpiración (Tr) y la evaporación (Ev), lo que permite distinguir entre el consumo de agua útil para el crecimiento del gasto no productivo; 2- El desarrollo del canopeo se efectúa a partir de un modelo que simula su crecimiento y senescencia a lo largo del ciclo, siendo la base para estimar Tr del cultivo y su separación de la Ev del suelo; 3- Estima el rendimiento final $(\mathrm{Y})$ en función de la biomasa acumulada $(\mathrm{B})$ y el índice de cosecha (IC); 4- Pondera el efecto del estrés hídrico sobre cuatro componentes distintos: crecimiento y senescencia de la canopia, Tr e IC.

En una fase operativa, AquaCrop presenta como ventajas la facilidad del manejo de datos de entrada, integrados por información meteorológica/climática de: suelo, prácticas agronómicas, a partir de una interfase configurada de manera muy amigable y fácil de utilizar. En maíz ha sido calibrado bajo distintos ambientes y condiciones de manejo, tanto en secano como bajo riego, mostrando un comportamiento promisorio si se tienen en cuenta las numerosas simplificaciones consideradas por Hsiao et al., (2009).

Las medidas matemáticas directas que conducen a realizar un análisis de crecimiento de una planta o un cultivo en particular en condiciones naturales 0 controladas de desarrollo fenológico y fisiológico han dado como resultado un análisis de crecimiento que ha sido usado ampliamente para el estudio de los factores que influencian el desarrollo de la planta y el rendimiento, a través del seguimiento de la acumulación de materia seca durante el tiempo (Santos et al., 2010).

La acumulación de materia seca por una población de plantas, depende principalmente del total de carbono fijado y obedece a la intercepción de radiación fotosintéticamente activa y su uso eficiente por el dosel del cultivo (Torres et al., 2012). La intercepción es determinada en primer lugar por el índice de área foliar 
(IAF) y por el coeficiente de extinción, un índice de eficiencia de intercepción de la radiación, mientras que la eficiencia fisiológica en el uso de esta radiación puede medirse de manera indirecta a través de la tasa de asimilación neta (TAN) que es la ganancia neta de asimilados por unidad de área foliar en un tiempo determinado. El IAF y la TAN son componentes de la tasa de crecimiento del cultivo (TCC) que mide la ganancia de materia seca por una población de plantas en una determinada área (Torres et al., 2012).

Estos componentes fisiológicos (IAF y TAN) son afectados por la absorción de agua y nutrientes por la raíz, y cuando existe estrés por alguno de estos factores; por ejemplo, por deficiencias nutricionales se puede afectar la captura de radiación y su eficiencia en la producción de biomasa (Tollenaar et al., 2006).

Debido a la diversidad de zonas productoras de maíz tecnificado, este documento presenta resultados del proceso de adaptación del modelo AquaCrop y la parametrización de diferentes variables para un cultivo de maíz bajo las condiciones de Altillanura, mediante un análisis de sensibilidad de algunos parámetros, basados en un seguimiento en campo observando el crecimiento y desarrollo del cultivo, permitiendo de esta manera discutir el efecto de las diferentes condiciones climáticas de temperatura, radiación y precipitación, en búsqueda de mejorar las consideraciones relacionadas con prácticas de manejo apropiadas, lograr un cultivo de maíz mejor adaptado a los procesos de clima, suelo, manejo agronómico y ambiente de la Altillanura colombiana.

\section{METODOLOGÍA}

\section{Condiciones del cultivo de maíz}

Este cultivo es de tipo comercial, hibrido BM709, siendo establecido en la vereda Chaviva de Puerto López Meta en el km 89 de la vía que de Villavicencio conduce al municipio de Puerto Gaitán Meta, enmarcado dentro del paisaje de Altillanura colombiana. Las coordenadas geográficas del sitio son: 4¹9’48.00 N, 72¹5’56.72” O y una altura de $185 \mathrm{msnm}$ (Figura 1). El clima de la zona es monomodal con una temporada de baja precipitación marcada, que se presenta entre los meses de 
Diciembre hasta Marzo y una época de alta precipitación entre los meses Abril hasta Noviembre, con precipitación total anual de 2200-2500 mm, temperatura media anual que oscila en un promedio de $27.4^{\circ} \mathrm{C}$ y los vientos con una velocidad promedio anual de $1.6 \mathrm{mt} / \mathrm{seg}$.

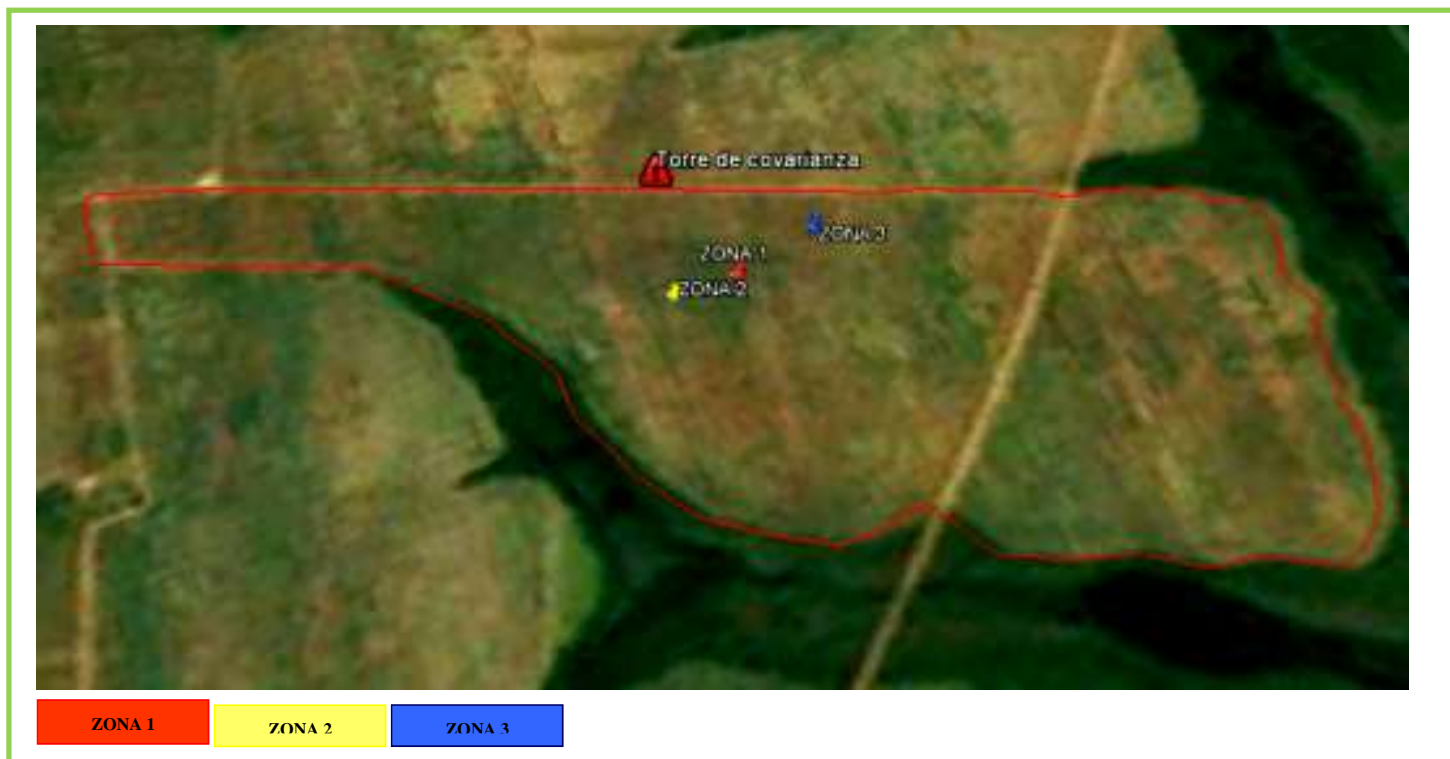

Figura 1. Ubicación geográfica del lote del maíz y distribución de cada una de las zonas de muestreo.

Con el propósito de realizar mediciones de crecimiento y comprender el comportamiento de los diferentes órganos de la planta en su acumulación de materia seca bajo las condiciones de Altillanura, se realizó la evaluación en un campo sembrado con maíz comercial hibrido BM709, que presenta un alto techo productivo, buena estabilidad y adaptación a las condiciones de ambiente y suelo de la Altillanura Colombiana. Se realizaron 10 muestreos para medidas biométricas en plantas fijas (Fotografía 1), donde se midieron cuatro (4) plantas, espaciadas a 5 metros la una de la otra, y que se encontraban repartidas equidistantemente en el lote en tres zonas abarcadas por el radio de $800 \mathrm{~m}^{2}$ de la torre de Eddy covariance (Fotografía 2) otros muestreos se hicieron al azar, tomando en cada uno la parte aérea a lo largo del ciclo del cultivo en cercanía a cada uno de los puntos fijos.

Los muestreos se finalizaron el día en que se realizó la cosecha total del lote que constaba de 154 ha, estas evaluaciones se iniciaron desde los 12 días después de 
la siembra en la fase vegetativa hasta el día 115 de grano lleno, los 9 muestreos se hicieron a los 12, 22, 29, 36, 43, 57, 71, 85 y 115 días después de la siembra. Rincón et al., (2007) definen que el crecimiento de los diferentes órganos de las plantas, es un proceso fisiológico complejo, que depende directamente de la fotosíntesis, la respiración, la división celular, la elongación, la diferenciación, entre otros, que además está influenciada por factores como temperatura, intensidad de luz, densidad de siembra, calidad de la semilla, disponibilidad de agua y de nutrientes.

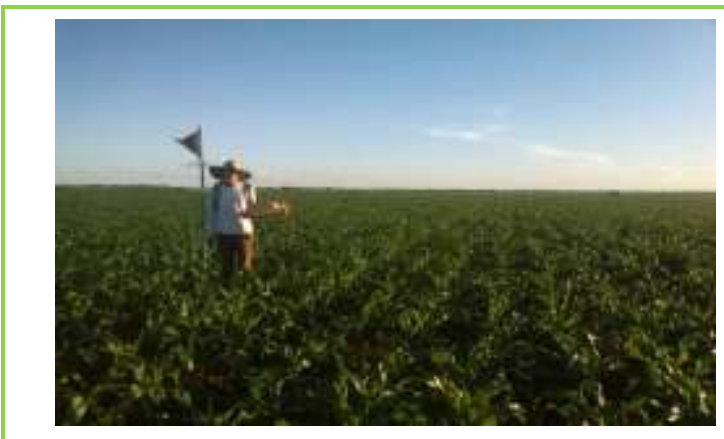

Fotografía 1. Punto de muestreo biométrico (Izquierda)

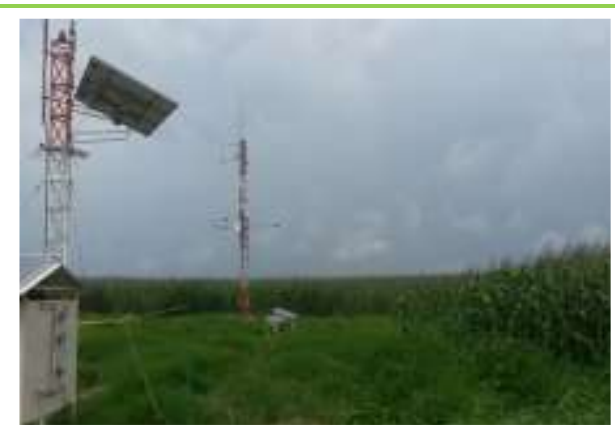

Fotografía 3. Torre de Eddy covariance y cultivo de maíz (derecha)

Para esta investigación se tuvieron en cuenta algunas medidas directas de crecimiento espaciadas a nivel del lote en tres zonas equidistantemente representativas respecto al radio de medición de la torre de Eddy covariance, cada una de estas medidas se generó tomando el número de plantas representadas en un metro lineal, con el propósito de determinar un patrón de variación entre los puntos y poder ser llevadas a un análisis de varianza (ANOVA) junto con las medidas biométricas, para poder simular el desarrollo del cultivo de las tres zonas definidas como uno solo y conocer de esta manera las posibles diferencias de desarrollo que se pueden presentar y así lograr estimar las condiciones de desarrollo del cultivo en función del tiempo como se describen a continuación.

\section{Estimación del desarrollo del cultivo}

1- Materia seca total de la planta: Con la población experimental establecida se comenzaron las mediciones de crecimiento de acuerdo a los anteriormente descritos, estableciendo un estado de desarrollo de las plantas evaluadas de 
acuerdo a la escala fenológica de la BBCH (Hack et al., 1992 ), se realizó el muestreo en un metro lineal en selección completamente al azar en cada una de las tres zonas definidas, tomando las estructuras aéreas de hoja, tallo, panoja y mazorca, para ser llevadas hasta materia seca en peso constante en una estufa de secado a $70^{\circ} \mathrm{C}$ durante $48 \mathrm{~h}$ y así conocer la distribución de biomasa durante el desarrollo del cultivo.

2- Área foliar: Para evaluar esta variable, se midió largo y ancho de todas las hojas de cada planta; multiplicándolo por 0.75 para determinar área foliar por hoja, lo que permitió obtener el total por planta y el correspondiente índice de área foliar dividiéndola entre el área del suelo ocupada por la planta, durante todas las etapas de crecimiento evaluadas, sabiendo que la población inicial sembrada fue 80.000 plantas por hectárea (área de cada planta $50 \mathrm{~cm} \times 25 \mathrm{~cm}$ ).

3- Índices de crecimiento: Hunt, (1990) establece que, que estos índices son utilizados para un análisis de crecimiento son: el IAF, entendido como el área de las hojas del cultivo presente en un metro cuadrado de suelo, siendo así, una medida que no tiene unidades; la TCC mide el incremento de la masa seca por unidad de tiempo y área $\left(\mathrm{g} / \mathrm{día} / \mathrm{m}^{-2}\right)$ y TAN que mide la acumulación de masa seca en función del área foliar por unidad de tiempo (Tabla 1). Dicha información fue analizada mediante el paquete estadístico Infostat se aplicó un análisis de varianza ANOVA para diferencias entre zonas y la prueba de Tukey $(P<0.05)$ a fin de poder realizar un análisis a las tres zonas con un único patrón medio de comportamiento.

\section{Modelo AquaCrop}

En el presente trabajo, se utilizó la versión 5.0 del AquaCrop, la cual fue adaptada a estas condiciones para mayor confiabilidad a las condiciones de la región (Figura 2). Para ser más exacta la estimación de las variables de estudio se dispuso de la siguiente información como: condiciones meteorológicas, suelos en cuanto a variables hidrofísicas en varios horizontes y rendimientos histórico del cultivo. La caracterización climática con los datos generados por la torre de Eddy covariance se realizó la adaptación del modelo de AquaCrop, durante el periodo en los cuales 
se tomarán datos de crecimiento y desarrollo del cultivo. Las variables climáticas tomadas fueron: precipitación (PPT), temperatura máxima ( $\mathrm{T}$ máx.), temperatura mínima (T mín.), Humedad relativa (HR), Brillo solar (Br. Solar) y Evapotranspiración de referencia del cultivo (ETo) la cual fue calculada mediante el software ETo Calculator, y que fueron ingresadas en el módulo de clima del software AquaCrop.

Tabla1. Índices fisiológicos de crecimiento y sus fórmulas

\begin{tabular}{|c|c|}
\hline Índice de crecimiento & Fórmula para su cálculo \\
\hline Tasa de acumulación neta & $\begin{array}{l}\text { TAN }=((\text { msf }- \text { msi }) /(\text { tf }- \text { ti })) \times((\text { In aff }- \text { In } \\
\text { afi }) /(\text { aff }- \text { afi }))\end{array}$ \\
\hline Índice de área foliar & $\mathrm{IAF}=\mathrm{af} / \mathrm{as}$ \\
\hline Tasa de crecimiento del cultivo & $\begin{array}{l}\text { TCC }=\{((\text { msf }- \text { msi }) /(\text { tf }- \text { ti })) \times((\text { In aff }- \text { In } \\
\text { afi }) /(\text { aff }- \text { afi }))\} \times(\text { af } / \text { as })\end{array}$ \\
\hline $\begin{array}{l}\text { Dónde: } \\
\text { msf = masa seca final } \\
\mathrm{msi}=\text { masa seca inicial } \\
\mathrm{tf}=\text { tiempo final (días) } \\
\mathrm{ti}=\text { tiempo inicial (días) } \\
\text { In msf = logaritmo natural de masa seca } \\
\text { final } \\
\text { In msi = logaritmo natural de masa seca } \\
\text { inicial }\end{array}$ & $\begin{array}{l}\text { aff = área foliar final } \\
\text { afi = área foliar inicial } \\
\text { In aff = logaritmo natural de área foliar final } \\
\text { In afi = logaritmo natural de área foliar } \\
\text { inicial } \\
\text { af = área foliar } \\
\text { as = área de suelo }\end{array}$ \\
\hline
\end{tabular}

Fuente: Adaptado de Rincón et al., (2007)

Dentro del modelo AquaCrop se tiene un grupo de módulos (Figura 3), que permiten ingresar las variables externas, de estado y los parámetros no Conservativos que son los propios de cada región.

Se utilizaron registros diarios la torre de Eddy covariance: precipitación, radiación, temperatura máxima y mínima, y evapotranspiración de referencia (ETo); concentración de $\mathrm{CO}_{2}$ asignada de manera aproximada dentro del modelo de AquaCrop los cuales fueron calculados por la torre en 413ppm. La evapotranspiración se estimó con el modelo ETo Calculator por medio de registros diarios de las variables de temperatura máxima y mínima, humedad relativa y radiación neta. A continuación, se describen la forma como se realizaron los ajustes de los tres módulos: Cultivo, suelo y manejo del cultivo. 


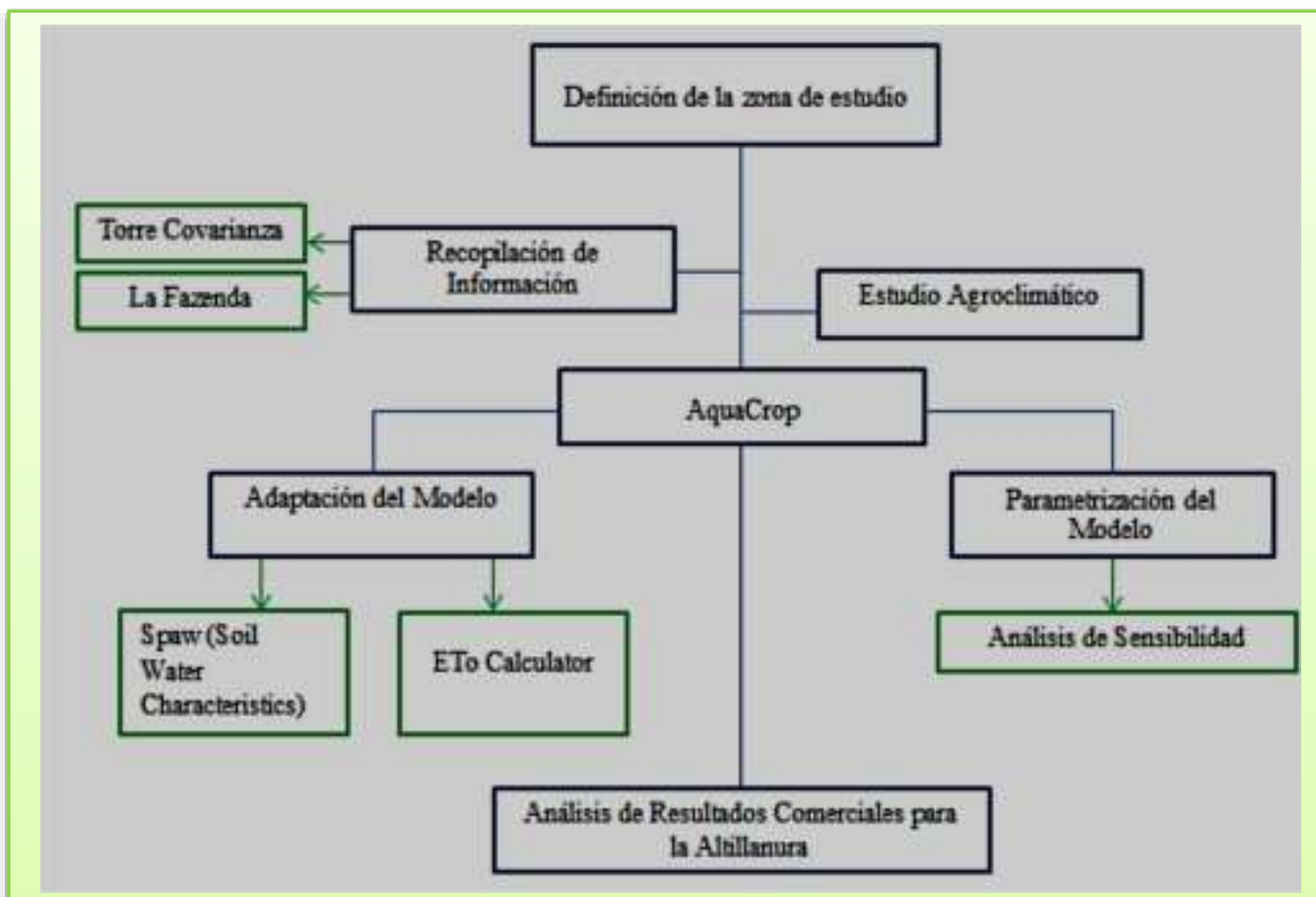

Figura 2. Diagrama metodológico para la parametrización y generación de resultados del AquaCrop.

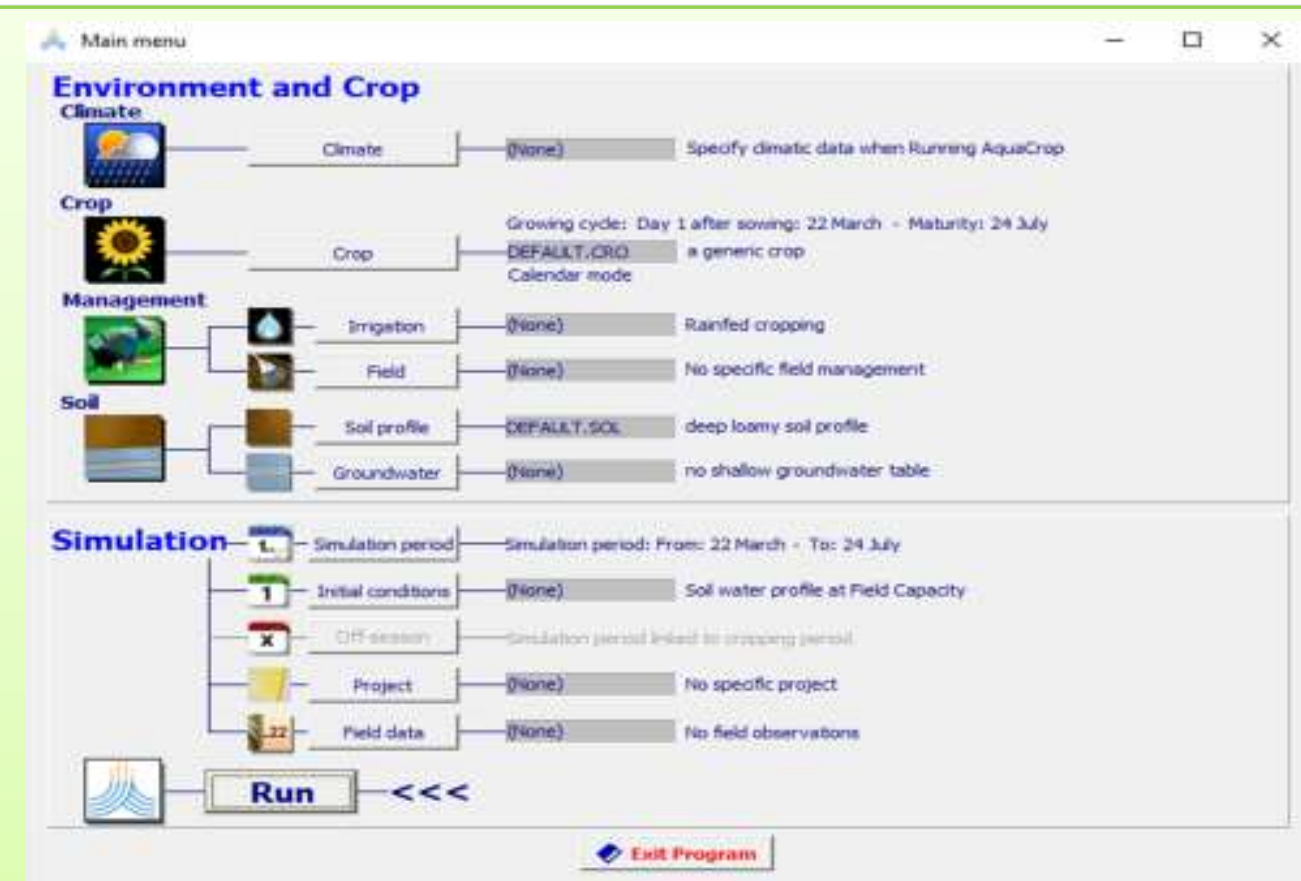

Figura 3. Ventanas de entrada de información para cada uno de los módulos de AquaCrop. 


\section{Módulo de cultivo}

Para el ajuste del modelo de AquaCrop se realizaron simulaciones, inicialmente sin modificar los parámetros "conservativos" del cultivo y se compararon las salidas en cuanto a rendimiento respecto a la información de campo. A su vez se evaluó estadísticamente el seguimiento de las variables biomasa seca, rendimiento del grano seco e índice de cosecha y follaje (Canopy Cover), durante el ciclo de cultivo apoyado en el análisis de crecimiento durante el ciclo del cultivo. Los Parámetros conservativos son específicos para cada cultivo que no cambian sustancialmente con el tiempo, las prácticas de gestión, la ubicación geográfica o el clima. También se supone que no cambian con cultivares a menos que se demuestre lo contrario. Se calibran con datos de la cosecha cultivados en condiciones favorables y no con limitantes, pero siguen siendo aplicables para situaciones de estrés a través de su modulación por funciones de respuesta (Steduto et al., 2012).

\section{Módulo suelo}

Se requirió información de parámetros físicos de textura y para punto de Marchitez permanente, capacidad de campo, punto de saturación y conductividad hidráulica saturada, se utilizó el software SPAW, en el módulo Soil Water Caharacteristics. Esta información se refirió a perfiles de calicatas medidas en campo en cercanías a los lotes bajo estudio.

\section{Módulo de manejo de cultivo}

Riego: Debido a la adecuada precipitación y poca área, donde se realizó el experimento, no fue necesaria la aplicación de riego.

Prácticas agrícolas: El modelo AquaCrop asume porcentaje de fertilidad del cultivo fijo durante todo el ciclo, el cual es una relación entre la biomasa observada y la potencial. De tal forma, se estimó el rango de fertilidad moderado (entre 59 y $75 \%$ ), el cual fue corroborado en campo por las medidas biométricas tomadas. 


\section{Ajuste del modelo de AquaCrop}

Para este ajuste del modelo en el material del cultivo de maíz seleccionado, se realizó Altillanura siguiendo un esquema (Figura 4), a partir de información recopilada por la torre de Eddy covariance para los datos de clima de la época, el cual tuvo como punto de partida la definición de los parámetros no conservativos, que se llevó a cabo en las distintas etapas del cultivo, de acuerdo a los registros de rendimientos de biomasa y producto cosechado, se logró observar el comportamiento de los diferentes procesos o variables determinantes que caracterizan el proceso evolutivo del cultivo y su resultado final: cobertura de follaje, biomasa, rendimiento. Estos parámetros fueron la base de la simulación realizada en el software, en tanto que estos valores son comparados con los resultados obtenidos en campo. Si el ajuste obtenido no es el adecuado se procedía a cambiar algún/algunos parámetros conservativos y se volvía a iniciar el proceso descrito. En el caso de estudio que se describe, el patrón de ajuste se determinó por medio del seguimiento de la materia seca llevada a cabo durante el análisis de crecimiento del cultivo. Se analizó el ajuste de acumulación de biomasa y rendimiento seco, en índice de cosecha, y evolución de cobertura. Se decidió cambiar el parámetro conservativo de fertilidad potencial para mejorar el ajuste a las condiciones de Altillanura, de acuerdo a los análisis de sensibilidad.

\section{Análisis de sensibilidad}

Para entender la susceptibilidad del modelo a los cambios en las características medioambientales y cómo esa susceptibilidad puede afectar las salidas en biomasa y/o rendimientos, con base en el ajuste para la Altillanura, se seleccionaron algunas de las principales entradas del modelo, siendo modificadas dentro de un rango específico para observar las alteraciones en la producción del cultivo. Todas las comparaciones de variación porcentual debieron ser respecto a los rendimientos finales en la etapa de ajuste del modelo. El análisis de sensibilidad se llevó a cabo sobre las siguientes entradas: nivel de fertilidad del suelo, comportamiento del modelo AquaCrop variando los índices de cosecha, el Canopy Cover, escenarios de $\mathrm{CO}_{2}$ y condiciones iniciales de suelo. 


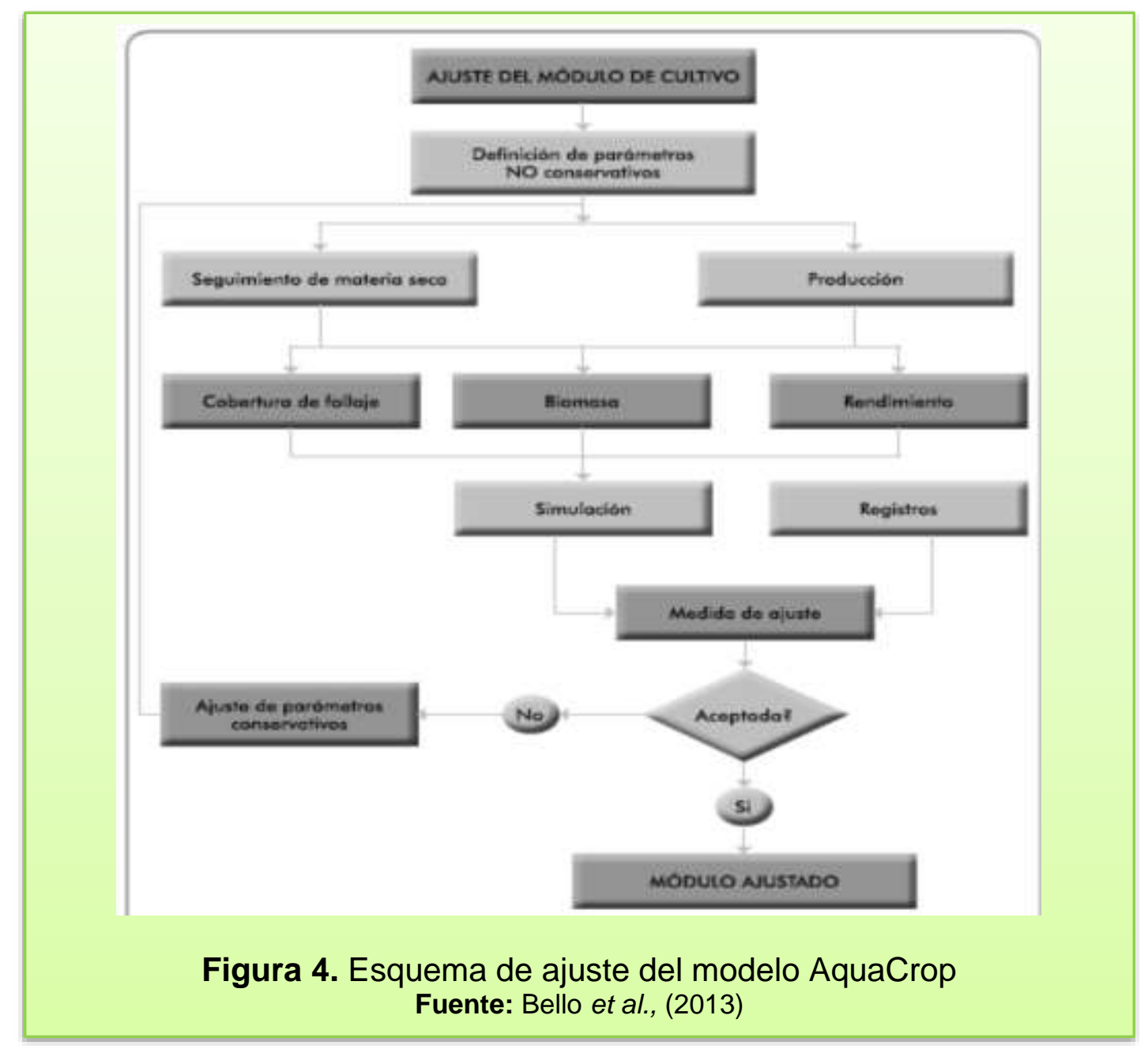

\section{RESULTADOS Y DISCUSIÓN}

Para realizar el seguimiento al crecimiento del cultivo de maíz se tuvo en cuenta los siguientes parámetros: fenología, área foliar, altura de planta, número de hojas y distribución de masa seca. De acuerdo con la metodología planteada se realizó una revisión de las medidas biométricas y tomadas durante cada una de las etapas de desarrollo referenciados dentro de la escalada de la $\mathrm{BBCH}$ (Tabla 2), estos datos fueron separados y tabulados para cada una de las zonas definidas en el lote, con el propósito de realizar una comparación entre los datos cada área y encontrar correlación entre los valores, que permitiera hacer un análisis de la información homogenizando estas tres zonas como un solo lote, en lo referente a la altura de planta, numero de hojas, acumulación de materia seca y área foliar, encontrando 
que para la variable de altura se presentaron diferencias entre zonas en los muestreos: dos, tres y cuatro, y para el de numero de hojas en la toma de muestra siete, mientras que para la acumulación de materia seca se observaron diferencias en la toma de datos: uno, cuatro y seis y para el área foliar en el muestreo número siete.

Tabla 2. Épocas de muestreo y estadio de desarrollo del cultivo de acuerdo a las escalas de $\mathrm{BBCH}$

\begin{tabular}{ccl}
\hline Muestreo & DDS & \\
\hline Uno & 3 & Estadio principal 0. Germinación \\
Dos & 12 & Escala BBCH \\
Tres & 22 & $\begin{array}{l}\text { Estadio principal 1. Desarrollo de las hojas (tallo } \\
\text { principal). }\end{array}$ \\
Cuatro & 29 & \\
Cinco & 36 & Estadio principal 3. Crecimiento longitudinal del tallo \\
principal
\end{tabular}

Escala fenológica de la BBCH (Kack, et al.,1992)

DDS = Días después de la siembra

De acuerdo a lo anterior, con el propósito de realizar un solo análisis de los datos se asumieron estas diferencias como errores en los coeficientes de variación, los cuales para ser datos de campo se encontraban bastante ajustados, para tomar las tres zonas como una sola y realizar el análisis al crecimiento y desarrollo del cultivo, bajo un desarrollo de cultivo modelado como un único lote. El resumen general de los datos finales arrojados por el análisis estadístico fue utilizado en el análisis del AquaCrop y en los cálculos de los diferentes índices de crecimiento, tomando las medias de la prueba estadística de cada uno de los resultados, para realizar los respectivos gráficos y análisis. 
Teniendo en cuenta que la biomasa total de un cultivo hasta su cosecha, es la fijación de carbono de las plantas durante todo su ciclo de crecimiento, la cual se ve afectada por diferentes factores climáticos, manejo agronómico, suelo, ambiente y material genético empleado, debido a la eficiencia con que las plantas utilizan cada uno de estos recursos (Figura 5), aclarando que cultivos eficientes como el maíz tienden a depositar la mayor parte de su crecimiento en aumentar su área foliar, para aprovechar la radiación solar neta en la fijación de carbono, por tanto la tasa de crecimiento de cultivo (TCC) de una especie está relacionada estrechamente a la intercepción de radiación solar, que para este cultivo presentó un declive a la edad de 29 días después de la siembra (DDS) y cuando la planta supero el índice de área foliar de cinco; así que la tasa de asimilación neta (TAN) (que se define como el aumento neto en peso seco por unidad de tiempo y por área foliar) es directamente proporcional al índice de área foliar (IAF), durante este periodo de los 22 a 29 días se genera un estancamiento en el aumento del IAF el cual hizo declinar la TCC, debido a factores climáticos que inhibieron la intersección de radiación por parte de las plantas perdiendo eficiencia en la utilización de esa energía en la acumulación de carbono (Figura 5).

Al presentarse un declive de la TCC luego a 43 días, tiempo durante el cual se inicia la floración y se alcanza el máximo el inicio de máximo IAF (el cual determina la disponibilidad de carbohidratos para cumplir las funciones de mantenimiento de la planta y llenado de grano); al ser este directamente proporcional a la TAN (esta es alta cuando las plantas son pequeñas y la mayoría de las hojas están expuestas a la luz solar directa), a medida que el cultivo crece y el índice de área foliar se incrementa, más y se aumenta el número de hojas que comienzan a sombrearse, causando una disminución de la TAN a media que el crecimiento progresa, permite definir de esta manera el IAF óptimo bajo estas condiciones en 5.29; esto se sustenta en el estudio en el que (Brougham, 1965) mostró que la TCC aumentó hasta un IAF de cinco, en donde el canopeo interceptó el 95 \% de la radiación solar incidente, este autor afirma que un IAF por encima de cinco cambia significativamente la TCC, por lo tanto el IAF optimo, es cuando alcanza la máxima 
TCC, puesto que está disminuyó a medida que el IAF se incrementaba más allá del óptimo.

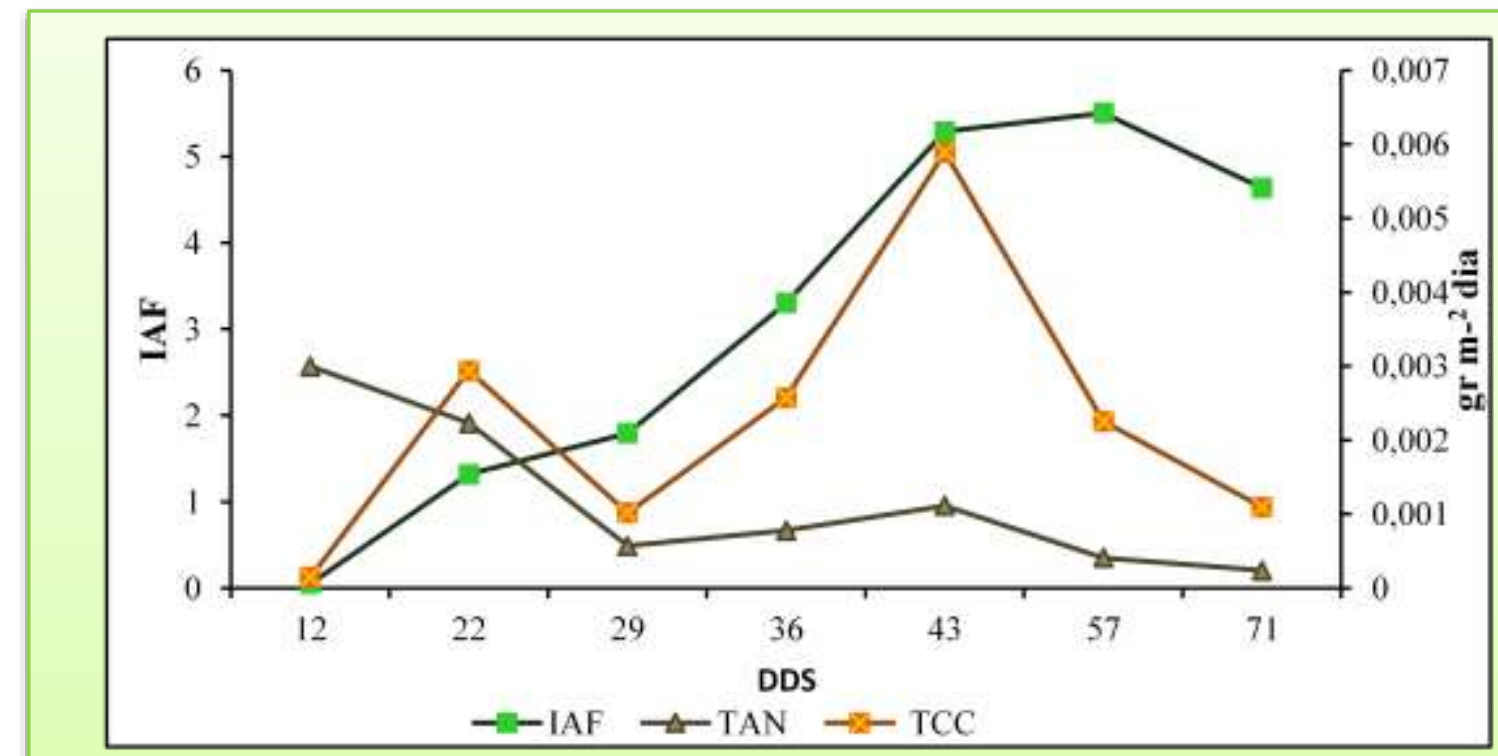

Figura 5. Índices que representan el comportamiento del crecimiento del cultivo de maíz.

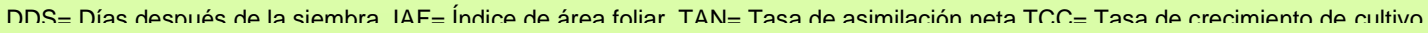

Este aumento significativo en la TCC en la fase vegetativa del cultivo, porque el IAF fue superior o igual a cinco, le permitiría a la planta desarrollar los procesos de acumulación de carbono para el inicio de la etapa de floración, para adelantar este proceso y de esta manera acumular en un mayor tiempo energía para el llenado de grano, ayudando a aumentar los rendimientos finales del cultivo. Es importante resaltar, que días antes al inicio de máximo índice de área foliar, se presentó una fertilización nitrogenada que ayudaría a elevar el número de hojas para aumentar la tasa de fotosíntesis, llevando a un incremento en la tasa de crecimiento del cultivo.

Andrade y Sadras, (2000) afirman que inicialmente el área foliar en maíz aumenta a una tasa exponencial, pero al comienzo es pequeña y la radiación interceptada no es significativa por varias semanas, mientras que en floración el desarrollo del área foliar, finaliza siendo el objetivo de las prácticas culturales maximizar la fotosíntesis del cultivo interceptando prácticamente toda la radiación solar incidente. 
Si se tiene en cuenta la importancia de medir la altura se puede determinar el grado de desarrollo del área foliar y el tamaño final de la planta en su función de captar la radiación necesaria que le permita acumular la cantidad de carbohidratos que requiere para el llenado de grano, el cual definirá su rendimiento, se puede observar que la altura media a los 29 días es superior a $38 \mathrm{~cm}$, con un decaimiento en la TCC en los primeros estadios, puesto que carece de superficie foliar para captar toda la luz, por lo tanto no es aprovechada la radiación para alcanzar un IAF y así se afecta su TCC. Posterior a ello, inicia un proceso de máxima elongación a los 36 días con un aumento de la radiación y de área fotosintetizante alcanzando una altura de 83 $\mathrm{cm}$, incrementado la acumulación de materia seca que continuará hasta la etapa reproductiva avanzada, lo que indica un proceso de alta elongación del tallo debido a que en la medida que despliega nuevas hojas, la proporción de radiación que es interceptada por el cultivo aumenta y con ella la TCC producto de haber alcanzado un IAF critico; esta TCC se eleva a medida que se incrementa la eficiencia de intercepción de radiación.

Toyer y Brown (1976), en la determinación de la producción, en algunos casos, el tamaño de una planta es más importante que la duración del período de llenado de grano, debido a que su rendimiento potencial en antesis está en función de su crecimiento previo porque que se desarrolla con menos competencia y su rendimiento potencial es más alto, en este caso previo al inicio de la floración la planta alcanzó una altura mayor a $147 \mathrm{~cm}$, llegando a ser casi el doble de su medida anterior y consolidando en esta etapa de floración el mayor número de hojas durante todo el ciclo de cultivo con 14 hojas.

Este incremento potencial en la altura de la planta finaliza con la antesis y es superior a $239 \mathrm{~cm} 57$ DDS, que coincide con una etapa de máximo número de hojas y un IAF optimo y que se continúa incrementando, lo cual genera un decaimiento en la TCC, por lo que la máxima acumulación de carbohidratos por para el llenado del grano ha cesado definiendo de esta manera el rendimiento final. Teniendo en cuenta que una vez cesada la TCC la planta solo creció $5.71 \mathrm{~cm}$ hasta el día de la cosecha. Es de aclarar que si se hubiese mantenido un IAF crítico (por debajo de 529) cuando 
el porcentaje de intercepción de radiación se aproximaba al 95\%, la TCC hubiese continuado y posiblemente al final esto hubiera generado un aumento en el rendimiento del grano.

Andrade et al., (1996) establecen que el crecimiento del cultivo resulta de la acumulación de biomasa vegetal, quedando determinado el rendimiento en grano de esa biomasa acumulada durante su crecimiento particionado entre los órganos de cosecha y el resto de la planta. La acumulación de biomasa se debe, principalmente, al balance neto positivo del intercambio de carbono entre la planta y su ambiente, donde las pérdidas por respiración son compensadas a través del proceso de fotosíntesis. Por lo tanto, la acumulación de biomasa en el cultivo depende de la cantidad de radiación solar disponible, de la capacidad del canopeo para interceptarla y de la eficiencia con que el cultivo convierte la radiación capturada en biomasa vegetal (Figura 6).

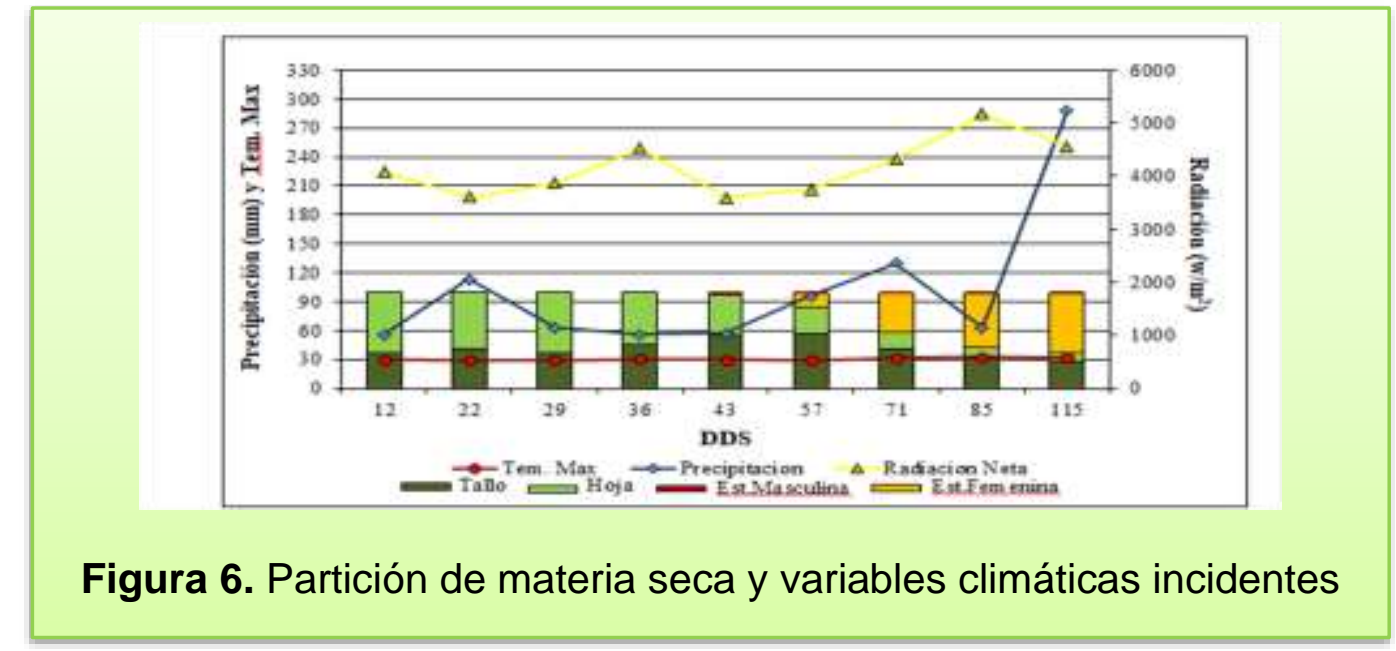

El seguimiento al desarrollo del cultivo en una precipitación total de $922.5 \mathrm{~mm}$, no se presentó limitantes por agua, considerando que Pedral et al., (2010) establece que los requerimientos hídricos del maíz durante su ciclo son alrededor de 550 a $575 \mathrm{~mm}$ con un óptimo de $800 \mathrm{~mm}$, inferior a lo reportado en el presente estudio. Es de aclarar que la mayor parte de estas precipitaciones ocurrieron en horas de la noche y madrugada, sin afectar las horas de mayor radiación del día donde la planta genera su mayor tasa fotosintética. 
Durante el desarrollo del cultivo la temperatura mínima y máxima fluctuaron entre 30 y $33^{\circ} \mathrm{C}$, y 22 y $23^{\circ} \mathrm{C}$, respectivamente, siendo sus promedios 22 a $33^{\circ} \mathrm{C}$, por lo que éstas no fueron limitantes para una mayor expresión del rendimiento del material de maíz, debido a que la temperatura máxima en la cual se afecta el desarrollo de este cultivo se encuentra entre 40 y $44^{\circ} \mathrm{C}$ (Kiniry y Bonhomme, 1991).

Para que un cultivo use eficientemente la radiación solar, gran parte de ésta debe ser absorbida por los tejidos fotosintéticos, siendo las hojas los órganos responsables de la fotosíntesis e intercepción de luz iniciando un desarrollo exponencial, aunque su capacidad de captar luz en sus primeras etapas se ve limitada por la cantidad de área foliar que empieza a ser determinante cuando esta no se alcanza un IAF crítico. Cultivos eficientes como los C4, entre ellos el maíz tienden a invertir la mayor parte de su crecimiento temprano en expandir su área foliar, lo que resulta en un mejor aprovechamiento de la radiación solar (Figura 6), las primeras etapas constituidas hasta los 29 DDS, la planta almacena cerca del $60 \%$ de su materia seca en las hojas. Una vez se inicia aumentos en la radiación, genera una máxima elongación y la distribución de sus carbohidratos, por lo que en canopeos con un alto IAF, las hojas jóvenes en la parte superior absorben la mayor proporción de radiación, tienen una alta tasa de asimilación de $\mathrm{CO}_{2}$ y translocan gran cantidad de compuestos hacia otras partes de la planta, a los 36 DDS hay una redistribución de estos asimilados y posteriormente al inicio de la floración hacia el tallo, a los 43 DDS.

A los 36 DDS con el aumento en los niveles de radiación y alcanzándose el IAF critico la planta comienza un rápido incremento en la acumulación de materia seca que continuará hasta la etapa reproductiva avanzada. Esto se debe a que las condiciones de temperatura durante el día llegaron en ocasiones a alcanzar valores de $35^{\circ} \mathrm{C}$, factor que afecta la tasa de crecimiento del cultivo, ocasionando que se modifique la duración de las distintas etapas ontogénicas del cultivo, afectando su crecimiento y consecuentemente el tiempo en el cual la radiación puede ser interceptada y transformada en materia seca, también influye el número final de hojas y el desarrollo del follaje, el cual define su IAF (Tollenaar et al., 2006), esto 
trajo como efecto un adelanto en la etapa de floración si se tiene en cuenta que se reportaba que la floración se a los 50 días, sin embargo se alcanzó a los 43 días en estas condiciones.

Andrade y Sadras, (2000) establecen que el inicio del mayor IAF encontrado a los 43 días coincidió con el inicio del desarrollo reproductivo de la planta, momento en el cual necesita de su máxima capacidad fotosintética para acumular carbohidratos y materia seca, para ser utilizados posteriormente en la formación de la mazorca, entre los 47 y 53 DDS, con capacidad de removilización de los carbohidratos de reserva a zonas que para esta investigación estuvieron dirigidos al tallo, estructuras reproductivas y finalmente la mazorca debido a que la planta alcanzo un IAF optimo que llevo a la disminución en su TCC.

Zamski y Schaffer (1996), establecen que la fracción del crecimiento total asignada a la formación del grano depende del patrón de repartición de la materia vegetal producida en la planta a lo largo de su ciclo de vida. Los granos constituyen los órganos de interés económico en maíz y por lo tanto, la fracción que se busca maximizar, en el porcentaje del peso total producido, el cual termina alojado en la mazorca y finalmente en los granos, alcanzando en esta investigación entre el 60 al $62 \%$.

Generalmente la densidad de siembra depende del genotipo, además de otras variables como disponibilidad de agua, manejos agronómicos, fertilidad del suelo, entre otros; por otro lado, la oferta de radiación solar debe ser la adecuada para la densidad de siembra definida, además es importante tener en cuenta que los vientos fuertes que se presentan en la región pueden causar problemas con materiales de porte muy alto los cuales pueden volcarse generando pérdidas en el rendimiento. Durante la época seca que se presenta en la zona, los pocos eventos de lluvia hacen que la evapotranspiración (ETo) sea alta, lo que lleva a que el déficit hídrico sea muy alto limitando la producción de maíz, o se disminuye ostensiblemente la producción de grano. 
En el ajuste del modelo AquaCrop se simularon las condiciones de suelo e índice de cosecha, la fertilidad del suelo usada fue del 100\% y el índice de cosecha se dejó por defecto en el $50 \%$, los parámetros de cultivo se introdujeron tal cual como lo refería la literatura del hibrido, en esta simulación los componentes de clima y suelo, otros factores como: horizontes, textura, y conductividad hidráulica, fueron medidos en campo. Esta simulación dio como resultado una productividad en grano de $15.718 \mathrm{Ton} / \mathrm{ha}$ y una biomasa seca de 31.4 Ton/ha; posteriormente se realizaron más corridas ajustando los valores a los medidos en el campo con el análisis de crecimiento y el resultado fue una biomasa de $22.4 \mathrm{Ton} / \mathrm{ha}$, utilizando el índice de cosecha medido en campo que fue del $53 \%$; dando una productividad en grano de 11.853 Ton/ha, para esta simulación se usó una fertilidad del suelo del $73 \%$,valor que se encuentra en un rango moderado de acuerdo al software, dada las condiciones de la zona y las buenas labores del cultivo se realizaron durante todo su ciclo bajo el sistema de mínima labranza con siembra directa.

Se presentó una reducción del $28.87 \%$ en la producción de biomasa simulada en los dos escenarios uno con condiciones óptimas y otro con las condiciones reales del campo, en cuanto a producción de grano se observó una reducción del $24.58 \%$, en los dos escenarios, dicha reducción es considerablemente alta en productividad. Esto hace suponer que si se generan condiciones de cultivo de maximización de recursos ambientales, manejo agronómico y clima en la zona se podría llegar a obtener rendimientos superiores a 12 toneladas

la comparación de los rendimientos reales medidos en campo, y los que fueron simulados con el software, los cuales no difieren mucho y se soportan en el coeficiente de Spearman de 0.99 muy cercano a 1, esto indica una fuerte relación entre los datos de campo y los simulados; estos valores demuestran que la parametrización del modelo AquaCrop en las condiciones de cultivo de la zona estudiada, explican de una manera muy aproximada el desarrollo de acumulación de biomasa seca en el cultivo de maíz en las condiciones de suelos y ambientales de la Altillanura colombiana (Tabla 3). 
Tabla 3. Comparación de rendimientos medidos en campo y los simulados por I AquaCrop

\begin{tabular}{ccccccc}
\hline $\begin{array}{c}\text { Biomasa } \\
\text { Real } \\
\text { Ton/ha }\end{array}$ & $\begin{array}{c}\text { Biomasa } \\
\text { Simulada } \\
\text { Ton/ha }\end{array}$ & $\begin{array}{c}\text { Coeficiente de } \\
\text { Determinación }\end{array}$ & $\begin{array}{c}\text { Rendimiento } \\
\text { Real } \\
\text { Ton/ha }\end{array}$ & $\begin{array}{c}\text { Rendimiento } \\
\text { Simulado } \\
\text { Ton/ha }\end{array}$ & $\begin{array}{c}\text { Coeficiente de } \\
\text { Determinación }\end{array}$ & $\begin{array}{c}\text { Coeficiente } \\
\text { de } \\
\text { Spearman }\end{array}$ \\
\hline 22.67 & 22.36 & 0.9839 & 10.85 & 11.85 & 0.99 & 0.99 \\
\hline
\end{tabular}

El coeficiente de determinación fue 0.9839 en la estimación de biomasa aérea en Ton/ha y el seguimiento de biomasa seca, es decir que el modelo es muy acertado a la hora de predecir el comportamiento del material BM709 en estas condiciones por los resultados que se observan en el seguimiento de la producción de biomasa en las diferentes etapas de muestreo, los cuales demuestran estar en concordancia: los datos simulados con los reales, lo que se logró ajustando la fertilidad del suelo a 73\%; los datos simulados de materia seca total hasta el día de cosecha fueron de 22.36 Ton/ha y los reales de 22.67 Ton/ha, observándose una diferencia de 0.31 Ton/ha, lo cual es una diferencia baja que marca la asertividad del modelo AquaCrop; por otro lado, el crecimiento vegetativo fue constante hasta cerca de los 43 días cuando se presenta la floración y cesa el crecimiento vegetativo e inicia la fase reproductiva y la formación de grano (Figura 7).

En cuanto al rendimiento la acumulación del grano seco (Figura 8), empezó cerca a los 57 días, y continuo un crecimiento hasta los 115 días, tiempo en el que se realizó la cosecha del grano y el cultivo se encontraba en su madurez fisiológica, se puede observar que los datos medidos en campo y los simulados por el software AquaCrop son muy similares, teniendo un coeficiente de determinación de 0.99 , es decir que el modelo simuló el rendimiento con un alto grado de exactitud, el rendimiento real que se midió en campo de siendo de 10.85 ton/ha Vs el simulado de $11.85 \mathrm{Ton} / \mathrm{ha}$, la diferencia fue una tonelada, dicho cambio se puede deber a que las plantas en la simulación no tienen ataques de plagas, enfermedades, problemas de germinación, o perdidas en la cosecha.

En el canopy cover (CC) real y simulado, se observaron algunas diferencias entre los datos tomados en campo y los que AquaCrop (Figura 9), esto se debió 
principalmente a la poca cantidad de muestreos de área foliar que se realizaron. Los datos simulados y los reales coincidieron en la época de mayor cobertura del follaje que fue cercana a los 43 días de la siembra, etapa en la cual el cultivo se encontraba en el inicio de la de floración, el crecimiento de la cobertura del dosel es constante hasta el llenado de grano donde empieza a disminuir en los datos de campo se puede observar que ocurre entre los 57 y 60 días. Por otro lado, el modelo no toma en cuenta enfermedades o plagas que puedan disminuir la cobertura del dosel. El coeficiente de determinación para el CC real y simulado, fue de 0.8162 aunque es cercano a uno, es bajo en comparación con los resultados que se obtuvieron con la biomasa, este es un parámetro que se debe tener en cuenta para futuras simulaciones con el software dado que para obtener datos más exactos es pertinente que se realicen más muestreos de cobertura de follaje o canopy cover, lo anterior, se observó en el ejercicio de simulación debido a que el dato de cobertura máxima del follaje afecta la salida de biomasa y de rendimiento del grano en el caso del cultivo de maíz.

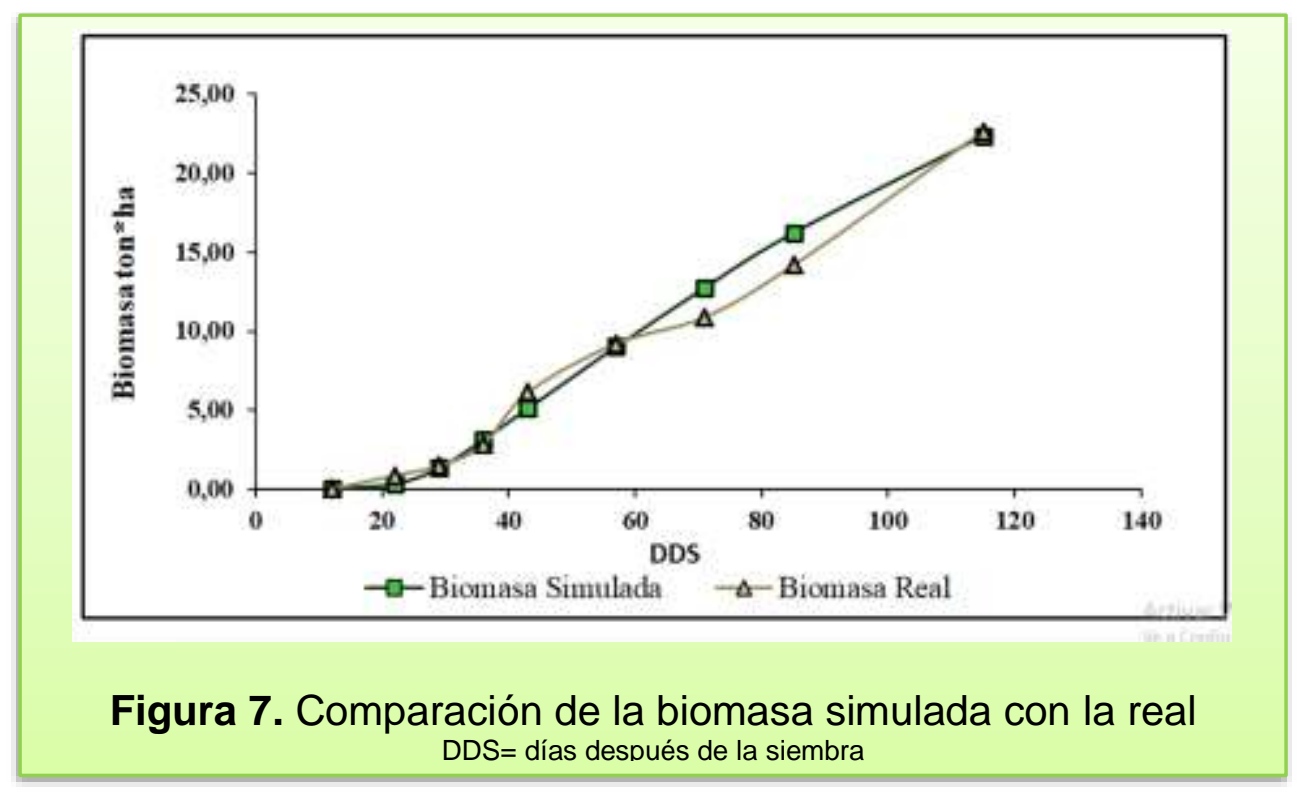

El software AquaCrop permite determinar las mejores fechas de siembra dependiendo de criterios ajustables de lluvia o de temperatura, logrando definir cuáles serían las fechas óptimas de siembra para obtener los mejores rendimientos. Es una ventana de oportunidad que se da para la siembra que es limitada, ya sea 
por factores climáticos o por disponibilidad de maquinaria y mano de obra, también permite saber cuál sería el rendimiento si se decide adelantar la siembra unos días o postergarlo. Aunque es muy importante tener en cuenta para los manejos de plagas y enfermedades que el software no tiene en cuenta esas variables.

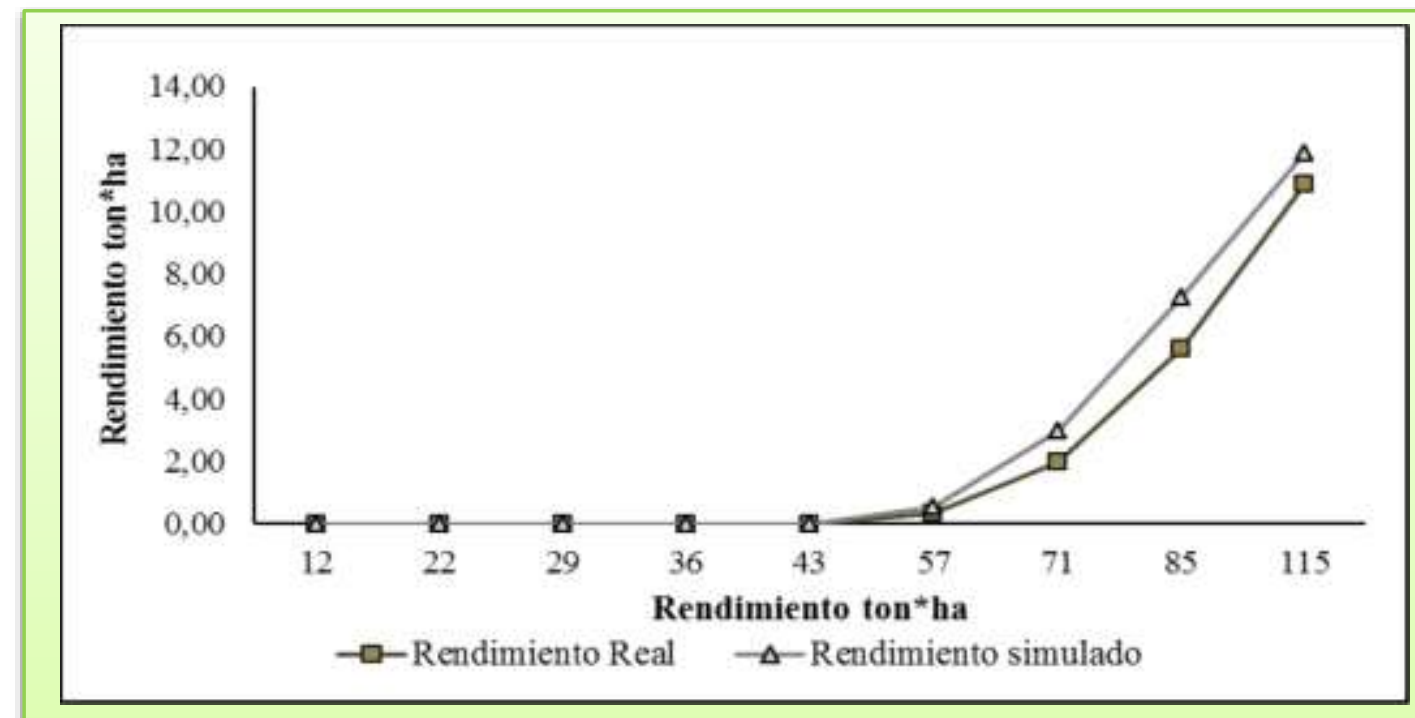

Figura 8. Seguimiento del rendimiento real y el simulado DDS= días después de la siembra

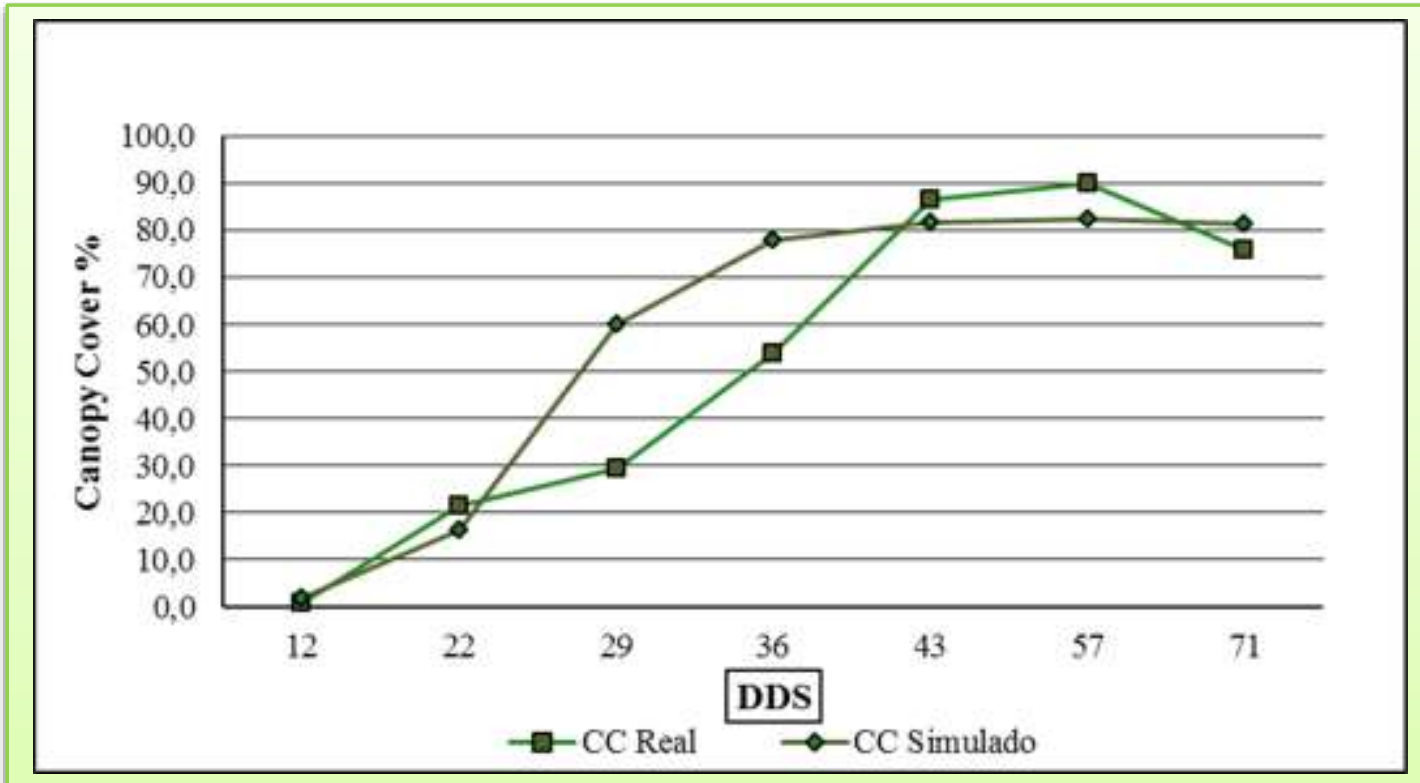

Figura 9. Canopy Cover (CC) con datos reales de campo comparado con el simulado por AquaCrop

DDS= días después de la siembra 
Al atrasar la siembra, las mayores temperaturas aceleran la velocidad de aparición y despliegue de las hojas determinando el rápido establecimiento de un canopeo eficiente para capturar radiación. Este efecto, sumado a los niveles crecientes de radiación solar incidente hacia el verano, le permite a las siembras tardías acumular una cantidad de radiación interceptada hasta la floración semejante a la de las siembras tempranas a pesar del menor número de días transcurridos. Las mayores temperaturas durante la fase vegetativa que experimentan las siembras tardías favorecen una alta eficiencia de conversión de luz en crecimiento (Andrade et al., 2000) acumulando generalmente más biomasa al momento de floración que las siembras tempranas.

La concentración de $\mathrm{CO}_{2}$ obtenida fue 415 ppm, con una fertilidad del suelo del $73 \%$, además se observó que la planta no tuvo otros factores de estrés que impactaran negativamente su rendimiento, porque había una buena disponibilidad de agua en el suelo, por las constantes precipitaciones que se presentaron en la zona (Figura 10), el ajuste de los datos de $\mathrm{CO}_{2}$ que trae por defecto el software, se logró obtener los niveles medios de este compuesto durante el ciclo de cultivo e ingresar el dato real de $\mathrm{CO}_{2}$ que fue de $415 \mathrm{ppm}$, este dato es muy importante para que el software simulara las condiciones de una forma precisa, puesto que concentración de $\mathrm{CO}_{2}$ en el ambiente determinan la tasa fotosintética del cultivo aunque algunos autores señalan que los cambios en plantas $C 4$ son bajos, según Salinger et al., (1997). El maíz presenta características fisiológicas favorables en lo que se refiere a la eficiencia de conversión de $\mathrm{CO}_{2}$ de la atmósfera, en compuestos orgánicos como los carbohidratos. Este proceso, que se realiza a través de la fotosíntesis, se refiere a la bioconversión de la energía solar en biomasa, en maíz la gran eficiencia de transformación de la energía luminosa en energía química, se debe al proceso fotosintético llamado "C4", en el cual, el $\mathrm{CO}_{2}$ es fijado en compuestos de cuatro carbonos; estos carbohidratos son continuamente almacenados en las células de la vaina vascular de hojas y posteriormente redistribuidos por toda planta. 


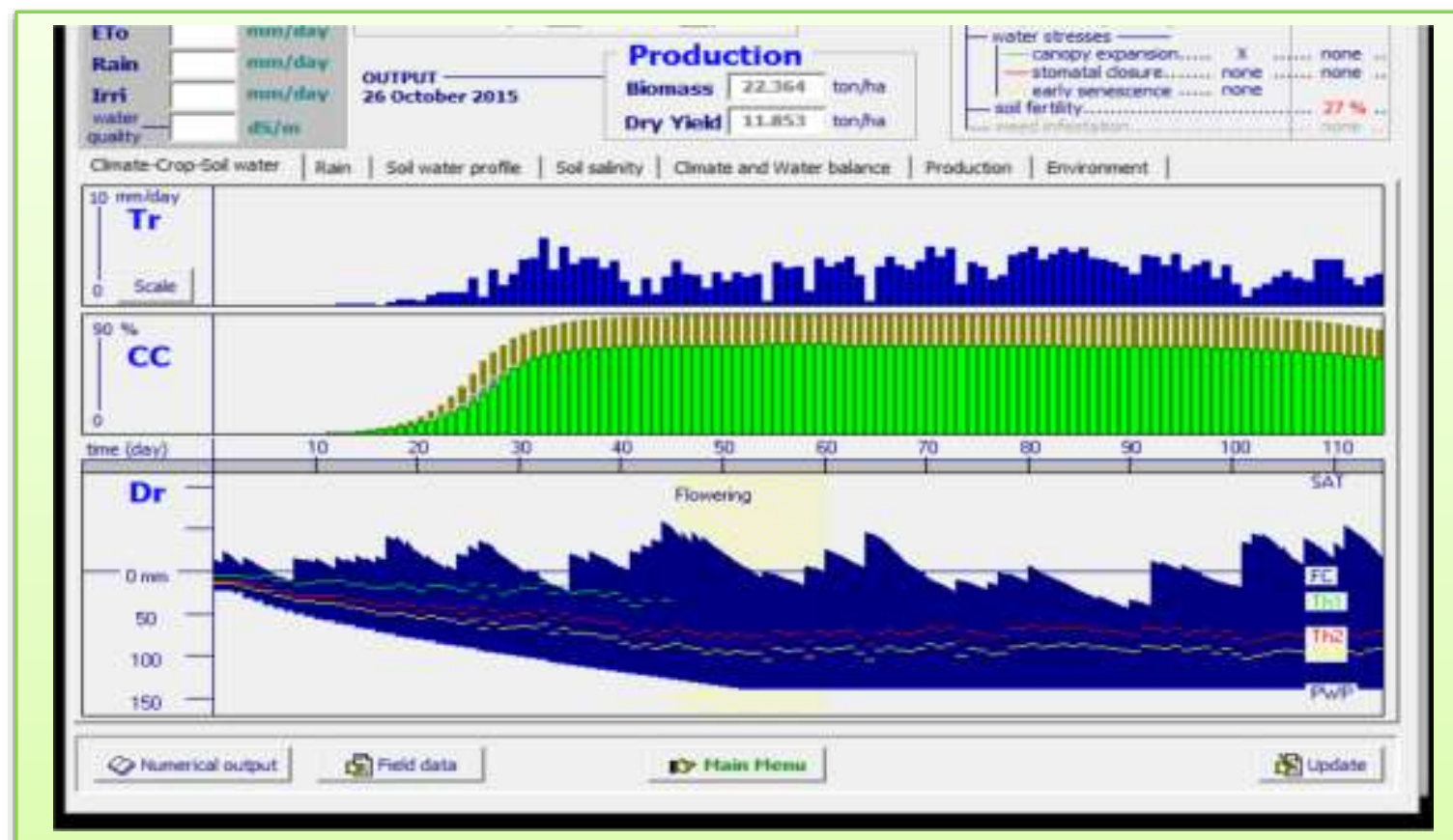

Figura 10. Salida de datos de la simulación con las condiciones propias de la Altillanura con el software AcuaCrop.

\section{CONCLUSIONES}

El análisis de desarrollo del cultivo en el software AquaCrop presento una representatividad entre lo simulado y lo real superior al $90 \%$ generando de esta manera un patrón de confiabilidad en la toma de decisiones referidas a maximizar los rendimientos del cultivo de maíz bajo las condiciones de la Altillanura.

El análisis al crecimiento y desarrollo logro mostrar que la producción total de materia seca es el resultado de la eficiencia cuando el cultivo intercepta y utiliza la radiación solar disponible durante la fase de crecimiento, la cual en condiciones de Altillanura es buena por los factores ambientales y climáticos, entre ellos el $\mathrm{CO}_{2}$, radiación, temperatura y precipitación.

En escenarios de cambio climático en la altillanura, temperaturas mayores a $40^{\circ} \mathrm{C}$, el cultivo de maíz se vería disminuido en sus diferentes etapas ontogénicas, solo en algunos casos, cuando las condiciones hídricas, radiación, manejo agronómico sean adecuadas el maíz podría mantener o aumentar su productividad por la alta 
concentración de $\mathrm{CO}_{2}$ en el ambiente y su capacidad de alcanzar una TCC eficiente bajo un IAF crítico.

\section{RECOMENDACIONES}

Se recomienda posterior a la floración mantener un IAF critico por debajo de 5.29, permitiendo de esta manera que la TCC aumente hasta un IAF de 5.2, en donde el canopeo intercepte el $95 \%$ de la radiación solar incidente, de lo contrario se cambiaría significativamente la TCC, y se disminuiría el almacenamiento de fotoasimilados que se distribuirían hacia el llenado de grano.

Es importante realizar seguimientos más detallados del crecimiento de los cultivos en la zona de Altillanura, con el fin de tener datos más exactos del crecimiento y desarrollo de la planta, además de realizar experimentos más controlados para determinar la acumulación de biomasa, índice de cosecha, y canopy cover, datos importantes durante el proceso de ajuste del modelo; es necesario contar en lo posible con información climática de primera mano y un análisis de suelos completo, con variables físicas bien definidas y reales.

El software AquaCrop es una herramienta muy interesante para ser implementada no solo en la zona de la Altillanura si no en regiones agrícolas del país, para determinar la productividad de los cultivos prediciendo condiciones climáticas futuras, teniendo en cuenta su variabiabilidad, con el propósito de hacer más eficiente el uso y consumo de agua en un cultivo de maíz.

\section{REFERENCIAS BIBLIOGRÁFICAS}

1. Andrade, F., Sadras, V. Bases para el manejo del maíz, el girasol y la soja (No. F01 INTA 16999). INTA, Buenos Aires, Argentina. EEA Balcarce. 2000.

2. Andrade, F., Cirilo, A., Uhart, S., Otegui M. Ecofisiología del cultivo de Maíz. Estación experimental Agropecuaria Balcarce, Buenos Aires: Editorial La Barrosa. 1996.

3. Bello, C, Patiño, J., Almanza, E, Monroy, J, Steduto, P, Mejías, P. Uso del modelo Aquacrop para estimar rendimientos para el cultivo del maíz en los departamentos de Córdoba, Meta, Tolima y Valle del Cauca. Colombia: FAO. 2013.

4. Béziat, P, Ceschia, E., Dedieu, G. Carbon balance of a three crop succession over two cropland sites in South West France. Agricultural and Forest Meteorology, 149: 1628-1645. 2009.

5. Cifuentes, J. G. Determinación de niveles económicos óptimos de uso de agua en la producción de maíz (Zea mays L.) usando el simulador de rendimientos desarrollado por la FAO AquaCrop Model. Zamorano, Honduras. 2010.

6. Corbin, K, Scoot D, Erandathie L, Schuh A. Assessing the impact of crops on regional CO2 fluxes and atmospheric concentrations. Tellus B, $62 \quad(5): \quad 521-532 . \quad 2010 . \quad$ Disponible En: http://www.tellusb.net/index.php/tellusb/article/view/16602 
7. Departamento Administrativo Nacional de Estadística (DANE). Maíz tecnificado en Colombia. Bogotá D.C. 2005.

8. Departamento Nacional de Planeación (DPN). Política para el desarrollo integral de la Orinoquia: Altillanura - Fase 1. (Documento CONPES 3797), Bogotá D.C.: 2014.

9. D’Andrea K.E., Otegui M.E., Cirilo A.G., Eyhérabide G. Genotypic Variability in Morphological and Physiological Traits among Maize Inbred Lines - Nitrogen Responses. Crop Science, 46: 1266-1276. 2006.

10. Etter, A., Sarmiento, A., Romero, M.H., Land use changes (1970-2020) and the carbon emissions in the Colombian Llanos. En: Ecosystem Function in Savvanas. Measurement and modeling at landscape to global scales. CRC Press, p. 383-402. 2010.

11. FENALCE. Índice Cerealistas primer semestre 2011. Bogotá D.C: Federación Nacional de Cultivadores de Cereales y Leguminosas. 2011.

12. Fernández, M. Efectos del cambio climático en el rendimiento de tres cultivos mediante el uso del modelo Aquacrop. FONADE, IDEAM y BID, Bogotá. 2013.

13. Hack H., Bleiholder H., Buhr L., Meier U., Schnock-Fricke E., Weber E., Witzenberger A. Einheitliche Codierung der ph"anologischen Entwicklungsstadien mono-und dikotyler Pflanzen Erweiterte BBCH-Skala, Allgemeine. Nachrichtenblatt des Deutschen Pflanzenschutzdienstes, 44: 265-270. 1992.

14. Hsiao, T, Heng, L, Steduto, P, Raes D, Fereres E. AquaCrop-The FAO crop model for predicting yield response to water: III. Model parameterization and testing for maize. Agron. J. 101: 448-459. 2009.

15. Kiniry J.R., Bonhomme R. Predicting maize phenology. In: Predicting crop phenology. Ed.T. Hodges. CRC Press. Boca Raton, Ann. Arbor. Boston, p 115-131. 1991

16. Montgomery, E.G. Correlation studies of com. Nebraska Agricultural Station Annual Report, Lincoln, v. 24, p.108-159. 1911.

17. Nelson, G. C., Rosegrant, M. W., Koo, J., Robertson, R., Sulser, T., Zhu, T., Lee, D. Cambio climático: el impacto en la agricultura y los costos de adaptación. IFPRI. 2009.

18. Ojeda, W, Flores, H, Sifuentes, E, Mejia, E., Flores, H. Simulación del rendimiento de maíz (Zea mays L.) en el norte de Sinaloa usando el modelo Aquacrop. AGROCIENCIA, 47(4), 347-359. 2013.

19. Quiroz, A., D. Marín. Evaluación de la asociación maízquinchoncho, con siembra escalonada y dos niveles de fertilización. I. Fenología y crecimiento. Agron. Trop. 50: 99-122. 2000.

20. Raes, D., Steduto, P, Hsiao, T, Fereres, E. Aquacrop-The FAO Crop Model to Simulate Yield Response to Water: II. Main Algorithms and Software Description. Journal of Agronomy, p 438-447. 2009.

21. Rincón, Á., Ligarreto, G., Sanjuanelo, D. Crecimiento del maíz y los pastos (Brachiaria sp.) establecidos en monocultivo y asociados en suelos ácidos del piedemonte llanero colombiano. Agron. Colomb, 25(2), 264-272. 2007.

22. Santos, M., Segura, M., Nú́stez, C. Análisis de crecimiento y relación fuente-demanda de cuatro variedades de papa (Solanum tuberosum L.) en el municipio de Zipaquirá (Cundinamarca, Colombia). Revista Facultad Nacional de Agronomía, Medellín, 63 (1): 5253-5266. 2010.

23. Saunders, M., Kansiimet, F., Jones, M. Agricultural encroachment: implications for carbon sequestration in tropical African wetlands. Global Change Biology, 18: 1312-1321. 2012.

24. Salinger, M.; Desjardins, R.; Jones, B.; Sivakumar, M.; Strommen, N.; Veerasamy, S.; Lianhai, W. Climate variability, agriculture and forestry: an update. World Meteorological Organization. WMO-841. GenevaSwitzerland. 51 p. 1997.

25. Steduto, P., Hsiao T, Raes D, Fereres E. AquaCrop-The FAO crop model for predicting yield response to water: I. Concepts and underlying principles. Agron. J.101: 426-437. 2009.

26. Paliwal R. El maíz en los trópicos: mejoramiento y producción; morfología del maíz tropical (en línea). Roma IT. FAO. 2001. Recuperado 18 Noviembre 2015. Disponible En: http://www.fao.org/docrep/003/x7650s/x7650s00.htm

27. Tollenaar, M., Deen W, Echarte L, Liu W. Effect of crowding stress on dry matter accumulation and harvest index in maize. Agronomy Journal, 98 (4): 930-937. 2006.

28. Toyer, A.F.; Brown, W.L. Selection for early flowering in corn: seven late synthetics. Crop Science, 16 (6): 767-773. 1976.

29. Torres, J, Moreno, G, Barón, F. Variabilidad del crecimiento y rendimiento del cultivo de maíz para choclo (zea mays I.) como respuesta a diferencias en las propiedades químicas del suelo en la sabana de Bogotá, Colombia. Rev. Fac. Nal. Agr. Medellín, 65 (2): 6579-6583. 2012.

30. Ulloa, A. Controlando la naturaleza: ambientalismo transnacional y negociaciones locales en torno al cambio climático en territorios indígenas en Colombia. Iberoamericana, 13 (49), 2013. 